\section{JTI}

JOURNAL OF

TRAUMA AND INJURY

\title{
Surgical Outcomes in Patients with Simultaneous Traumatic Brain and Torso Injuries in a Single Regional Trauma Center over a 5-Year Period
}

\author{
Jung-Ho Yun, M.D. \\ Department of Neurosurgery, Trauma Center, Dankook University Hospital, Cheonan, \\ Korea
}

Received: December 12, 2021

Purpose: The purpose of the study is to analyze the results of surgical treatment of patients with brain and torso injury for 5 years in a single regional trauma center.

Methods: We analyzed multiple trauma patients who underwent brain surgery and torso surgery for chest or abdominal injury simultaneously or sequentially among all 14,175 trauma patients who visited Dankook University Hospital Regional Trauma Center from January 2015 to December 2019.

Results: A total of 25 patients underwent brain surgery and chest or abdominal surgery, with an average age of 55.4 years, 17 men and eight women. As a result of surgical treatment, there were 14 patients who underwent the surgery on the same day (resuscitative surgery), of which five patients underwent surgery simultaneously, four patients underwent brain surgery first, and one patient underwent chest surgery first, four patients underwent abdominal surgery first. Among the 25 treated patients, the 10 patients died, which the cause of death was five severe brain injuries and four hemorrhagic shocks.

Conclusions: In multiple damaged patients require both torso surgery and head surgery, poor prognosis was associated with low initial Glasgow Coma Scale and high Injury Severity Score. On the other hand, patients had good prognosis when blood pressure was maintained and operation for traumatic brain injury was performed first. At the same time, patients who had operation on head and torso simultaneously had extremely low survival rates. This may be associated with secondary brain injury due to low perfusion pressure or continuous hypotension and the traumatic coagulopathy caused by massive bleeding.

Keywords: Multiple trauma; Brain injuries; Treatment outcome; Hypotension; Torso (http://creativecommons.org/licenses/by-nc/4.0/) which permits unrestricted noncommercial use, distribution, and reproduction in any medium, provided the original work is properly cited. 


\section{INTRODUCTION}

Traumatic collision at high speed frequently causes multiple body lesions and severe damage to various specific internal structures, including major life-supporting organs, such as the brain, heart, lungs, and intestines. Among these injuries, traumatic brain injury (TBI) is a leading cause of mortality and a sequela of trauma $[1,2]$. In approximately one third to half of cases, TBIs are accompanied by severe extracranial injury (ECI) in the chest, abdomen, and extremities $[3,4]$. Many authors have reported that head injury accompanied by severe ECI causes mortality at high rates $[5,6]$. However, the outcome of TBI is not particularly affected by extracranial factors $[7,8]$. TBI management itself, from initial surgical plan to postoperative intensive care unit care, is complicated. In addition, it is much more difficult to treat TBI in patients with concurrent injuries to the lungs, heart, abdominal organs, or other internal structures.

Most of the known publications about TBI with ECI so far have focused on only the overall results of treatment for TBI and have not covered surgical treatment of ECI. Also, in most studies, ECI has included injuries to the pelvis, extremities, and neck, in addition to severe chest and abdominal injuries. Hemorrhages on the face, neck, and extremities are compressible and can be controlled quickly, whereas hemorrhages involving the head, chest, and abdomen are not compressible and are usually more complicated internal injuries that necessitate urgent surgical treatment. In this study, Ifocused on the outcomes of surgical treatment of life-threatening abdominal, chest, and pelvic injuries in patients who underwent the surgery either simultaneously for brain and torso injuries or sequentially over a 5-year period in a single regional trauma center in South Korea.

\section{METHODS}

This retrospective study was approved by the Institutional Review Board (IRB) of Dankook University Hospital (IRB No. DKUH 202112028).

\section{Patient population and clinical data}

A total of 14,175 patients who suffered trauma were admitted to Dankook University Hospital Regional Trauma Center, a regional trauma center, between January 2015 and December 2019. We focused on the cases of 25 consecutive patients with severe TBI who underwent operations for brain injury and torso hemorrhage, including chest or abdomen injury. We excluded patients who underwent surgery for organ donation or simple procedures such as tracheostomy or wound management. We defined TBI as severe when a patient's initial Glasgow Coma Scale (GCS) score was 3-8, when the head Abbreviated Injury Scale (AIS) score was 4-5 (including subdural score of 4-5, epidural score of 5 , or intracerebral hematoma score of 4-5), or when decompressive craniectomy was necessary. Clinical information was obtained through a retrospective review of each patient's medical records. We also documented each patient's demographic data, AIS score, Injury Severity Score (ISS), initial GCS score, initial vital sign measurements (blood pressure, heart rate, and respiration rate), and Revised Trauma Score (RTS), and we investigated various data for treatment results, including mortality rate, preventable trauma death rate (PTDR), and Glasgow Outcome Scale (GOS) score 3 months after injury.

\section{Surgical treatment strategy}

In accordance with the Advanced Trauma Life Support (ATLS) guidelines of the American College of Surgeons, we conducted the primary and secondary surveys after initial resuscitation of patients at Dankook University Hospital Regional Trauma Center and then planned appropriate treatment. In cases of severe TBI and abdominal injuries that necessitated emergency surgery, various factors such as blood pressure, neurological signs, and radiologic findings were used to prioritize injuries, and in cases of severe TBI and thoracic injuries, vital signs such as blood pressure, heart rate, and respiration rate were also considered. Simultaneous brain and abdominal or thoracic surgery was performed only in patients with poor vital signs in whom brain surgery was inevitable because of worsening neurological symptoms. 


\section{Statistical analysis}

To analyze the correlation of each factor with the occurrence of complications, we performed univariable regression analysis, using the chi-squared test or Fisher's exact test, along with SPSS Statistics 20.0 (IBM Corporation, Armonk, NY, USA). $p$-values of $<0.05$ were considered statistically significant.

\section{RESULTS}

\section{Patients' characteristics}

The demographic characteristics of the 25 patients (17 men and eight women) are summarized in Table 1. All patients underwent brain surgery and thoracic or abdominal surgery; their average age was 55.4 years. The mean ISS was 34.7 (range 9-59), the mean initial GCS score was 9 (range 3-15), and the mean initial systolic blood pressure (SBP) was $125 \mathrm{mmHg}$ (range $0-227 \mathrm{mmHg}$ ). The causes of injury included 14 traffic accidents, 10 falls, and one crushing by heavy machinery. Fourteen patients underwent surgery on the same day as the injury, of whom five patients underwent simultaneous surgery for TBI and ECI, four patients underwent brain surgery first, one patient underwent chest surgery first, and four patients underwent abdominal surgery first. Ten patients (40\%) died during treatment: 5 from severe brain injury, four from hemorrhagic shock, and 1 from multiorgan failure. Of the 15 survivors, seven patients had a good prognosis according to their GOS scores $(\geq 4)$. Among the patients who underwent multiple surgeries, significant factors associated with mortality were lower SBP, higher initial heart rate, lower GCS score, and higher ISS. In addition, the higher the head AIS score, the more likely a patient was to die. However, age, initial RTS, and initial hemoglobin level were not associated with mortality.

\section{Surgical results}

We evaluated the 14 patients with TBI and torso hemorrhage who underwent resuscitative operations (Table 2).

Table 1. Clinical characteristics of enrolled patients and risk factors associated with mortality

\begin{tabular}{|c|c|c|c|c|}
\hline & Total $(n=25)$ & Survival group $(n=15)$ & None survival group $(n=10)$ & $p$-value \\
\hline Age (years) & $55.4(19-78)$ & $56(29-78)$ & $57.6(19-78)$ & 0.40 \\
\hline Male & 17 & 10 & 7 & 0.38 \\
\hline \multicolumn{5}{|l|}{ Mechanism } \\
\hline Traffic accident & 14 & 9 & 5 & \\
\hline Fall down & 10 & 5 & 5 & \\
\hline Heavy object & 1 & 1 & 0 & \\
\hline \multicolumn{5}{|l|}{ Initial V/S } \\
\hline $\mathrm{SBP}(\mathrm{mmHg})$ & 125 & 130 & 116 & 0.081 \\
\hline Heart rate (/min) & 96 & 91 & 106 & 0.02 \\
\hline Respiration rate (/min) & 19 & 22 & 14 & 0.13 \\
\hline Mean initial GCS & $9(3-15)$ & $11(5-15)$ & $6.3(2-15)$ & $<0.001$ \\
\hline ISS & $34.7(9-59)$ & $29(9-50)$ & $43(24-59)$ & $<0.001$ \\
\hline Head AIS & 4.2 & 3.9 & 4.6 & $<0.001$ \\
\hline Chest AIS & 3.3 & 3.2 & 3.5 & 0.12 \\
\hline Abdomen AlS & 2.8 & 2.6 & 2.9 & 0.15 \\
\hline RTS & $9(0-12)$ & $10(9-12)$ & $7(0-12)$ & 0.062 \\
\hline $\mathrm{Hb}$ & $11.7(2-15.2)$ & $12.4(9.4-15.2)$ & $10.8(2-14.3)$ & 0.52 \\
\hline
\end{tabular}

Values are presented as mean (range).

V/S: vital sign, SBP: systolic blood pressure, GCS: Glasgow Coma Scale, ISS: Injury Severity Scale, AIS: Abbreviation Injury Scale, RTS: Revised Trauma Scale, $\mathrm{Hb}$ : hemoglobin. 
Operations for TBI included decompressive craniectomy and hematoma evacuation in 10 patients and external ventricular drainage in four patients. Some patients with higher ISS and GCS score underwent surgery for ECI before surgery for TBI because their vital signs were unstable; in this group, the mortality rate was significantly lower $(25 \%)$ than in other groups, and none of the deaths were preventable. Conversely, in patients who had low

Table 2. Clinical data according to the resuscitative operation group (multiple surgery group at the same day: sequntial or simultaneous) depends on the sequnce of the surgery

\begin{tabular}{|c|c|c|c|c|}
\hline & $\begin{array}{l}\text { Total } \\
(n=14)\end{array}$ & $\begin{array}{l}\text { Simultaneous group } \\
\qquad(n=5)\end{array}$ & $\begin{array}{l}\text { ECI followed by TBI } \\
\text { group }(n=4)\end{array}$ & $\begin{array}{c}\text { TBI followed by ECI } \\
\text { group }(n=5)\end{array}$ \\
\hline Age (years) & $59.8(20-78)$ & $55.6(20-69)$ & $49(33-68)$ & $71(59-78)$ \\
\hline Male & 17 & 10 & 7 & 0.38 \\
\hline linitial GCS & $7.2(3-15)$ & $5.6(3-13)$ & $9.8(6-14)$ & $7.8(4-15)$ \\
\hline ISS & $39.5(24-59)$ & $42(34-50)$ & $42.3(38-45)$ & $38.2(24-45)$ \\
\hline \multicolumn{5}{|l|}{ Initial V/S } \\
\hline $\mathrm{SBP}(\mathrm{mmHg})$ & 108 & 128 & 121 & 99 \\
\hline Heart rate (/min) & 90 & 114 & 98 & 75 \\
\hline Respiration rate (/min) & 16 & 12 & 22 & 18 \\
\hline ISS $>35$ & $11(79.0 \%)$ & $4(80.0 \%)$ & $4(100.0 \%)$ & $3(60.0 \%)$ \\
\hline Good prognosis (GOS >4, 5) 3 months & $2(14.0 \%)$ & $0(0.0 \%)$ & $1(25.0 \%)$ & $1(2.00 \%)$ \\
\hline Morality (GOS 1) & $9 / 14(65.0 \%)$ & $5 / 5(100.0 \%)$ & $1 / 4(25.0 \%)$ & $3 / 5(60.0 \%)$ \\
\hline Preventable trauma death & $1 / 9(11.0 \%)$ & 1 (MODS) & 0 & 0 \\
\hline
\end{tabular}

Values are presented as mean (range).

ECl: extracranial injury, TBl: traumatic brain injury, GCS: Glasgow Coma Scale, ISS: Injury Severity Scale, V/S: vital sign, SBP: systolic blood pressure, AIS: Abbreviation Injury Scale, GOS: Glasgow outcome, MODS: multiorgan dysfunction syndrome.

Table 3. Statistical analysis for extracranial injury group according to the body lesion of the surgery

\begin{tabular}{|c|c|c|c|c|}
\hline & $\begin{array}{c}\text { Total } \\
(\mathrm{n}=25)\end{array}$ & $\begin{array}{l}\text { TBI wit thorax injury: TT } \\
\qquad(n=6)\end{array}$ & $\begin{array}{l}\text { TBI with abdomen injury: } \\
\text { TA }(n=19)\end{array}$ & $p$-value \\
\hline Age (years) & $55.4(19-78)$ & $57(19-69)$ & $56(20-78)$ & 0.38 \\
\hline Male & 17 & 10 & 7 & 0.38 \\
\hline linitial GCS & $9(3-15)$ & $11.3(4-15)$ & $8.2(3-15)$ & $<0.001$ \\
\hline ISS & $34.7(9-59)$ & $30.5(17-50)$ & $36(9-59)$ & 0.004 \\
\hline \multicolumn{5}{|l|}{ Initial V/S } \\
\hline $\mathrm{SBP}(\mathrm{mmHg})$ & 125 & 126 & 123 & \\
\hline Heart rate (/min) & 96 & 94 & 97 & \\
\hline Respiration rate (/min) & 19 & 21 & 18 & \\
\hline ISS $>35$ & $13(52 \%)$ & $2(33 \%)$ & $11(58 \%)$ & 0.07 \\
\hline Good prognosis (GOS >4, 5) 3 months & $7(28 \%)$ & $3(50 \%)$ & $4(21 \%)$ & 0.057 \\
\hline Morality (GOS1) & $10 / 25(40 \%)$ & $2 / 6(33 \%)$ & $8 / 19(43 \%)$ & 0.35 \\
\hline Preventable trauma death & $1 / 10(10 \%)$ & 0 & 1 (MODS) & 0.58 \\
\hline
\end{tabular}

Values are presented as mean (range).

TBI: traumatic brain injury, TT: TBI with thorax injury, TA: TBI with abdomen injury, GCS: Glasgow Coma Scale, ISS: Injury Severity Scale, V/S: vital sign, SBP: systolic blood pressure, AIS: Abbreviation Injury Scale, GOS: Glasgow Outcome, MODS: multiorgan dysfunction syndrome. 
GCS scores (because of low blood pressure) who underwent surgery for ECI before surgery for TBI because of unstable vital signs, the injuries were no more severe than those of other groups, but the mortality rate was very high (60\%). In addition, among the patients who underwent TBI and ECI surgeries simultaneously, the overall injuries were extremely severe (mean ISS 42), the vital signs were unstable, and the level of consciousness was poor (mean initial GCS score 5.6), and so the prognosis was very poor; the mortality rate was $100 \%$.

Patients with TBI and ECI were classified as those with thoracic injury (the TT group) and those with abdominal injury (the TA group). Surgical results for these groups are summarized in Table 3. In comparison with the TA group, the TT group had a significantly lower mean ISS $(p=0.004)$ and a significantly higher mean initial GCS score $(p<0.001)$. The patient's treatment outcomes did not differ statistically, but $50 \%$ of the TT group and only $21 \%$ of the TA group had GOS scores of $\geq 4$, and the PTDR was a patient in the TA group.

\section{DISCUSSION}

Severe TBI is a major cause of death and disability [2]. Secondary brain injury caused by various extracranial factors is very common $[2,4]$. Establishing a reliable prognosis early after injury is notoriously difficult but necessary, as noted in the Hippocratic aphorism "No head injury is too severe to despair of, nor too trivial to ignore". The purpose of neurosurgical intervention is to immediately remove mass effects in the cranial cavity before irreversible brain stem damage or overall ischemic damage in the whole brain can occur. However, the prognosis of patients with severe TBI not only is subject to clinical symptoms and anatomical conditions of brain injury on admission but also is affected by the presence of systemic injuries that affect intracranial pressure and cerebral perfusion pressure.

Hypotension and coagulopathy can worsen the secondary effects of TBI, and thus not only the brain but also the whole body, including systemic circulation and coagulation, must be a focus of treatment of TBI and ECI [9]. Various therapeutic strategies, including hypo- thermia, have been attempted to improve the outcome of head trauma, despite the lack of definitive evidence of their effectiveness. Ho et al. [7] analyzed various factors in trauma-related mortality and concluded that hypotension (SBP $<90 \mathrm{mmHg}$ ) and hypoxemia (partial pressure of arterial oxygen $<60 \mathrm{mmHg}$ ) on admission were inadequate as indicators of extracranial injuries; by assessing ECI more comprehensively with the ISS to identify standard neurologic prognostic factors, they improved the accuracy of predictions of mortality after neurotrauma.

In particular, patients with TBI and other injuries may be at greater risk for adverse effects of such combined or interactive processes because TBI alone is inherently complex pathophysiologically and increases the risk for disability or mortality [10]. Secondary effects of TBI include neuroinflammation, excitotoxicity, metabolic disturbances, apoptosis, ischemia, oxidative stress, and blood-brain barrier disruption. For patients with multiple traumatic injuries, the systemic effects of significant ECI can exacerbate the secondary effects of TBI, particularly the neuroinflammatory response. Possible extracranial trauma-induced influences on TBI include elevated levels of circulating inflammatory cytokines, growth factors, and reactive oxygen species; for patients with bone fracture, fat emboli and mobilized mesenchymal stem cells increase the risk for morbidity. Moreover, multiple traumatic injuries may induce sepsis, systemic inflammatory response syndrome, and hemorrhagic shock [11].

Because of the combination of multiple traumatic injuries, treatment of patients with TBI and ECI is very complicated. Numerous supportive methods have been introduced to optimize treatment in such patients. The first treatment goal is to normalize brain tissue oxygenation and cerebral perfusion pressure. In patients who have sustained multiple traumas that could result in major blood loss, such as hemoperitoneum, hemothorax, or major fracture of the pelvis or extremities, physicians must choose from among numerous therapeutic methods to maintain and optimize mean arterial pressure and cerebral perfusion pressure. In this situation, volume status and cardiac contractibility are main indicators for systemic treatment such as fluid resuscitation or administration of inotropic or diuretic drugs [12]. Results of a study of patients with TBI and hemorrhagic shock who under- 
went resuscitation suggested that raising systemic blood pressure with vasopressin during initial fluid resuscitation yields no benefit in the presence of TBI and hemorrhagic shock with uncontrolled internal hemorrhage. Those results also indicated that establishing hemostasis should be a priority during resuscitation of a patient with multiple traumatic injuries [13].

Two major studies, Corticosteroid Randomization after Significant Head Injury (CRASH) [4] and International Mission for Prognosis and Analysis of Clinical Trials (IMPACT) [14], focused solely on factors related to brain injury; other extracranial injuries were not evaluated. Gennarelli et al. [15] showed that in comparison with patients with severe ECI but without TBI, those with both severe ECI and TBI had a threefold higher mortality rate. They also found that even in the absence of severe ECI, the mortality rate among patients with TBI was 1.5 times higher than among those with ECI alone. Conversely, according to a study focusing on treatment, intensive care in a nonneurosurgical center was associated with a twofold increase in risk for death, which suggests that such treatment settings are suboptimal [5]; thus, patients with severe TBI should be treated at trauma centers that are capable of immediate neurosurgical intervention.

The most comprehensive study to date of the influence of concomitant extracranial injuries on mortality rates in patients with TBI is a meta-analysis of the IMPACT, CRASH, and the Trauma Audit and Research Network databases, conducted by van Leeuwen et al. [16]. By analyzing adjusted integrated odds ratios for main prognostic parameters, including age, GCS score, pupil reactivity, and hypotension, the authors of this large observational study provided strong evidence that primary ECI (AIS score $<3$ ) increases mortality in patients with TBI. However, the effects of extracranial damage are less consequential with severe TBI than with mild and moderate TBI. In other words, the conclusions derived from this meta-analysis are similar to those derived from other large-scale trauma studies, and the degree of peripheral damage has a significant effect on mortality in patients with mild to moderate TBI but not in those with severe TBI.

Another study of the effect of ECI on patients with TBI demonstrated that multiple organ trauma and traumatic lung injury may worsen the outcomes in patients suffer- ing from severe TBI but are not significant predictors of mortality in this patient population [8]. However, like the study by van Leeuwen et al. [16], this study demonstrated that the more severe the brain injury was, the less the extracranial factors were likely to affect mortality.

Other predictors associated with mortality include age, RTS, and ISS [17-19]. Older age, lower RTS, and higher ISS were associated with a worse prognosis in patients with TBI. Important factors commonly mentioned in studies of TBI are the presence and duration of hypotension and hypoxia. In the presence of both TBI and ECI, injury or dysautoregulation of the brain renders patients more vulnerable to secondary insults such as hypotension or hypoxia and coagulopathy. In patients with TBI and ECI, the laboratory values related to coagulation other than platelet count were clearly abnormal. Thrombocytopenia, prothrombin time, and D-dimer abnormalities are independent risk factors of cerebral hypoxia or ischemia after injury and are associated with poor outcome after head trauma [20].

\section{Surgical strategy for patients with multiple traumatic injuries}

The treatment strategy for isolated TBI is completely different from that for TBI with multiple injuries. In patients with multiple injuries, the selection of which injury to treat first influences the outcome. If the vital signs of the patient are stable, surgical treatment can focus on the TBI; if not, the priority of surgical treatment is always to stabilize blood pressure and tissue oxygen saturation. This is because no matter how severe a TBI is, aggressive surgical treatment cannot be performed if the vital signs are unstable. Furthermore, persistent hypotension caused by ongoing hemorrhage worsens neurologic outcomes.

Several authors have discussed the strategy of simultaneous surgery when priorities cannot be determined. Lee et al. [21] proposed collaboration between neurosurgeons and surgeons from other departments for multiple life-threatening traumatic injuries. They stated that when serious damage to other organs is accompanied by severe TBI, active concurrent surgery can save a patient's life. To prevent secondary brain injury caused by hypotension, hypoxia, and hypercapnia, surgeons must determine why vital signs are unstable and then correct the problem rap- 
idly because the prognosis of patients with poor head AIS scores and low GCS scores depends on how early optimal function is restored [22].

Our findings are preliminary because information about surgical outcomes in patients who had severe TBI with severe ECI is sparse. Survival rates were higher among patients who underwent chest and brain surgeries than among those who underwent abdominal and brain surgeries. We presume that thoracic injury is less dangerous than abdominal injury and also more responsive to early resuscitative therapy, including fluid therapy, transfusion, and vasopressor or inotropic medication. Also, patients with severe thoracic injury are often excluded from studies because by the time they arrive at the hospital, resuscitation is often unsuccessful.

For patients with severe TBI who require definitive surgical intervention for massive bleeding in the chest or abdominal organs, hypotension and hypoxia must be addressed first; the next most important concern is the severity of neurological defects. In patients in very critical condition with severe hypotension, hypoxia, and neurological deficits, the treatment results are often poor when different types of surgery are performed simultaneously. In such cases, we recommend performing sequential surgery, with a short interval between procedures, starting with correction of hypotension and hypoxia (Fig. 1), because, as stated previously, if brain surgery is performed in the setting of hypotension and hypoxia, secondary brain injury will occur. General surgeons and neurosurgeons must collaborate during this process to optimize the outcome [23].

\section{Limitations}

This study had several limitations. First, it was a retrospective single center study with a small number of cases. Second, surgical treatment was limited to stopping bleeding and lowering intracranial pressure; subordinate procedures such as angiographic embolization, resuscitative endovascular balloon occlusion of the aorta, tracheostomy, and wound debridement surgery were excluded. Third, our evaluation of PTDR was limited to a single center. Further studies are needed to assess the long-term outcomes in a larger population of patients with both TBI and torso hemorrhage.

\section{CONCLUSION}

This study is the first in which outcomes of surgery in patients with brain and torso injuries were analyzed. In pa-
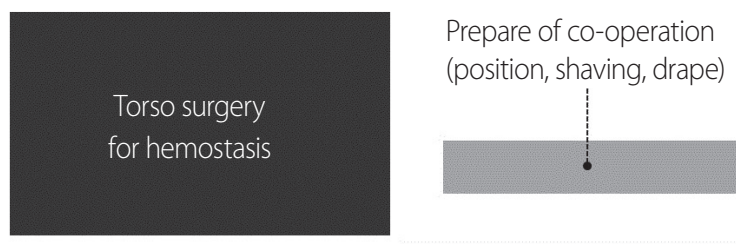

Start the surgery for the main bleeding focus

Stop the bleeding
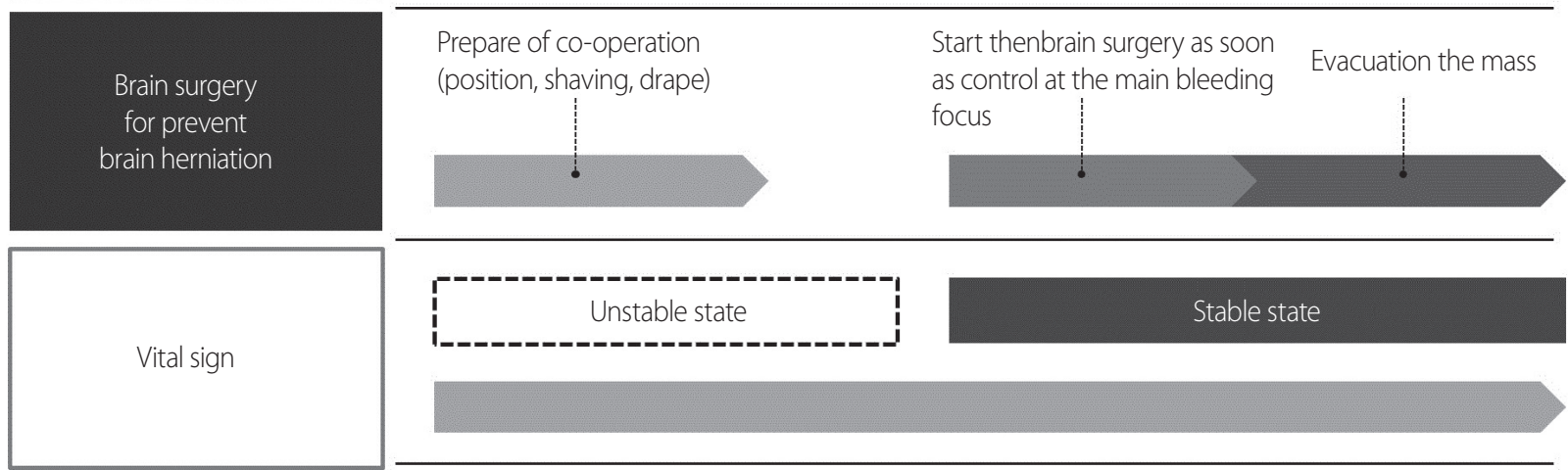

Unstable state

Stable state

Fig. 1. The timing of modified sequential operations in the Dankook University Hospital Trauma Center. Surgeons prepared for both torso and brain surgeries; the torso surgery was performed first to correct hypotension and hypoxia by stopping the bleeding, and brain surgery was performed immediately afterwards. A main indication that brain surgery may begin is the stabilization of vital signs. 
tients with multiple injuries who required abdominal or thoracic surgery in addition to brain surgery, low initial GCS score, and high ISS were associated with a poor prognosis. Patients with secondary brain injury that resulted from continuous hypotension or low perfusion pressure generally had a poor prognosis. Conversely, patients who had stable vital signs and were thus able to undergo brain surgery first had a better prognosis. Among patients who underwent surgery for both TBI and torso injuries simultaneously, the survival rate was extremely low. This may have been a result of secondary brain injury caused by low perfusion pressure or continuous hypotension, as well as the traumatic coagulopathy caused by massive bleeding.

\section{CONFLICTS OF INTEREST}

No potential conflict of interest relevant to this article was reported.

\section{INFORMED CONSENT}

This type of study does not require informed consent.

\section{ACKNOWLEDGEMENTS}

This work received 1st place best oral presentation award of pan pacific trauma congress 2021.

Author appreciates the help of traumatology professor Dong Hun Kim and Sung Wook Chang in Dankook University Hospital Trauma Center to present comments and shear the contents.

\section{REFERENCES}

1. Gennarelli TA, Champion HR, Sacco WJ, Copes WS, Alves WM. Mortality of patients with head injury and extracranial injury treated in trauma centers. J Trauma 1989;29:1193-201; discussion 1201-2.

2. Shackford SR, Mackersie RC, Holbrook TL, Davis JW, Hollingsworth-Fridlund P, Hoyt DB, et al. The epidemiology of traumatic death. A population-based analysis. Arch Surg 1993;128:571-5.

3. Leitgeb J, Mauritz W, Brazinova A, Majdan M, Wilbacher I. Impact of concomitant injuries on outcomes after traumatic brain injury. Arch Orthop Trauma Surg 2013;133:659-68.

4. MRC CRASH Trial Collaborators, Perel P, Arango M, Clayton T, Edwards P, Komolafe E, et al. Predicting outcome after traumatic brain injury: practical prognostic models based on large cohort of international patients. BMJ 2008;336:425-9.

5. Patel HC, Bouamra O, Woodford M, King AT, Yates DW, Lecky FE; Trauma Audit and Research Network. Trends in head injury outcome from 1989 to 2003 and the effect of neurosurgical care: an observational study. Lancet 2005;366:1538-44.

6. McMahon CG, Yates DW, Campbell FM, Hollis S, Woodford M. Unexpected contribution of moderate traumatic brain injury to death after major trauma. J Trauma 1999;47:891-5.

7. Ho KM, Burrell M, Rao S. Extracranial injuries are important in determining mortality of neurotrauma. Crit Care Med 2010;38:1562-8.

8. Baum J, Entezami P, Shah K, Medhkour A. Predictors of outcomes in traumatic brain injury. World Neurosurg 2016;90:5259.

9. Watanabe T, Kawai Y, Iwamura A, Maegawa N, Fukushima H, Okuchi K. Outcomes after traumatic brain injury with concomitant severe extracranial injuries. Neurol Med Chir (Tokyo) 2018;58:393-9.

10. Blennow K, Hardy J, Zetterberg H. The neuropathology and neurobiology of traumatic brain injury. Neuron 2012;76:88699.

11. McDonald SJ, Sun M, Agoston DV, Shultz SR. The effect of concomitant peripheral injury on traumatic brain injury pathobiology and outcome. J Neuroinflammation 2016;13:90.

12. So JS, Yun JH. The combined use of cardiac output and intracranial pressure monitoring to maintain optimal cerebral perfusion pressure and minimize complications for severe traumatic brain injury. Korean J Neurotrauma 2017;13:96-102.

13. Dickson JM, Wang X, St John AE, Lim EB, Stern SA, White NJ. Damage control resuscitation supplemented with vasopressin in a severe polytrauma model with traumatic brain injury and uncontrolled internal hemorrhage. Mil Med 2018;183:e460-6.

14. Maas AI, Marmarou A, Murray GD, Teasdale SG, Steyerberg EW. Prognosis and clinical trial design in traumatic brain injury: the IMPACT study. J Neurotrauma 2007;24:232-8.

15. Gennarelli TA, Champion HR, Copes WS, Sacco WJ. Compari- 
son of mortality, morbidity, and severity of 59,713 head injured patients with 114,447 patients with extracranial injuries. J Trauma 1994;37:962-8.

16. van Leeuwen N, Lingsma HF, Perel P, Lecky F, Roozenbeek B, $\mathrm{Lu}$ J, et al. Prognostic value of major extracranial injury in traumatic brain injury: an individual patient data meta-analysis in 39,274 patients. Neurosurgery 2012;70:811-8; discussion 818 .

17. The Brain Trauma Foundation. The American Association of Neurological Surgeons. The joint section on neurotrauma and critical care. Trauma systems. J Neurotrauma 2000;17:457-62.

18. Hukkelhoven CW, Steyerberg EW, Rampen AJ, Farace E, Habbema JD, Marshall LF, et al. Patient age and outcome following severe traumatic brain injury: an analysis of 5600 patients. J Neurosurg 2003;99:666-73.

19. Flaada JT, Leibson CL, Mandrekar JN, Diehl N, Perkins PK, Brown AW, et al. Relative risk of mortality after traumatic brain injury: a population-based study of the role of age and injury severity. J Neurotrauma 2007;24:435-45.

20. Chen H, Xue LX, Guo Y, Chen SW, Wang G, Cao HL, et al. The influence of hemocoagulation disorders on the development of posttraumatic cerebral infarction and outcome in patients with moderate or severe head trauma. Biomed Res Int 2013;2013:685174.

21. Lee HR, You NK, Seo SJ, Choi MS. Concurrent surgery of craniectomy and splenectomy as initial treatment in severe traumatic head injury: a case report. Korean J Neurotrauma 2017;13:141-3.

22. Stocchetti N, Furlan A, Volta F. Hypoxemia and arterial hypotension at the accident scene in head injury. J Trauma 1996;40:764-7.

23. Fukai J, Tsujimoto T, Yoshimura R, Raimura M, Kuwata T, Hyotani G, et al. Timing of craniotomy in a patient with multiple trauma including head injury. Neurol Med Chir (Tokyo) 2009;49:22-5. 